\title{
Effects of the conjugation of whey proteins with gellan polysaccharides on surfactant-induced competitive displacement from the air-water interface
}

\author{
B. Cai and S. Ikeda ${ }^{1}$ \\ Department of Food Science, University of Wisconsin, Madison 53706
}

\begin{abstract}
Whey proteins can be used to stabilize foams and emulsions against coalescence because of their ability to form viscoelastic films at the interface that resist film rupture on collision between colloidal particles. However, whey proteins are competitively displaced from the interface if small-molecule surfactants are added, leading to destabilization of the entire system. This is because surfactants are more effective in molecular packing at the interface, and they lower interfacial tension to a greater degree than whey proteins do, but their interfacial films are poor in viscoelasticity. We hypothesized that whey proteins would become more resistant to surfactant-induced competitive displacement if they were conjugated with network-forming polysaccharides. The protein moiety of the conjugate would be expected to enable its adsorption to the interface, and the polysaccharide moiety would be expected to form self-assembled networks, strengthening the interfacial film as a whole. In this study, whey proteins were conjugated with gellan polysaccharides using the Maillard reaction. Atomic force microscopy images of interfacial films formed by the whey protein-gellan conjugate at the air-water interface and transferred onto mica sheets using the Langmuir-Blodgett method revealed that gellan did form self-assembled networks at the interface and that interfacial films also contained a large number of unconjugated whey protein molecules. Following the addition of a small-molecule surfactant (Tween 20) to the sub-phase, surface pressure increased, indicating spontaneous adsorption of surfactants to the interface. Atomic force microscopy images showed decreases in interfacial area coverage by whey proteins as surface pressure increased. At a given surface pressure, the interfacial area coverage by whey protein-gellan conjugates was greater than coverage by unconjugated whey proteins, confirming that whey proteins became more resistant to surfactant-induced displacement after
\end{abstract}

Received December 15, 2015.

Accepted May 1, 2016.

${ }^{1}$ Corresponding author: shinya.ikeda@wisc.edu conjugation with gellan. Furthermore, gellan molecules added to the sub-phase after the formation of a monolayer of whey proteins at the air-water interface did not adsorb to the interfacial protein film. These results provide a molecular basis for designing interfacial structures to enhance the stability of colloidal systems. Key words: whey protein, gellan, conjugate, foam, emulsion

\section{INTRODUCTION}

Whey proteins can act as stabilizers for colloidal systems such as foams and emulsions, because their amphiphilic nature allows them to spontaneously adsorb to the air-water or oil-water interface and form viscoelastic interfacial films (Dickinson, 2001; Damodaran, 2005). Such films resist rupture, leading to coalescence on collision between colloidal particles. In contrast, the viscoelastic properties of interfacial films formed by small-molecular-weight surfactants are poor (Roth et al., 2000). Therefore, protein-stabilized colloidal systems are generally more stable. Furthermore, the elastic modulus of the interfacial film from the major whey protein $\beta-L G$ has been reported to be greater than that of $\beta$-CN (Mackie et al., 2001). The higher viscoelastic character of the interfacial film of the $\beta$-LG can be attributed to higher packing density and stronger protein-protein interactions at the interface, compared with the loose packing and weaker proteinprotein interactions of the much more flexible casein molecules (Dickinson, 2001).

The critical bulk concentration of whey proteins at which the interfacial tension reaches a plateau is much lower than that of small-molecule surfactants because of the much larger adsorption energy of whey protein molecules (De Feijter et al., 1987). However, at relatively high bulk concentrations, interfacial tension is lowered to a greater degree by surfactants than by whey proteins, because surfactants are more effective in molecular packing at the interface (Dickinson, 2001; Damodaran 2005). Consequently, as surfactant concentrations increase, whey proteins are competitively displaced from the interface. An orogenic displacement 
mechanism has been proposed based on atomic force microscopy (AFM) studies to explain such surfactantinduced competitive displacement (Mackie et al., 1999; Woodward et al., 2004, 2010a). The initial interfacial whey protein film contains void spaces due to packing limitations, allowing small-molecule surfactants to adsorb into these spaces, and then these nucleated sites expand, driven by the difference in surface pressure between surfactant and protein domains. As a result, the continuous protein film is laterally compressed, leading to an increase in film thickness. At sufficiently high surface pressures, the continuous protein film fails, and protein molecules finally desorb into the bulk phase.

An implication of the orogenic displacement mechanism is that the resistance of interfacial protein films to surfactant-induced competitive displacement can be improved by increasing its mechanical strength to make it more resistant to lateral compression (Morris et al., 2011). Dickinson and Hong (1994) treated $\beta-$ LG with heat before preparing an oil-in-water emulsion, and they found that the heat-treated whey protein was more resistant to displacement from Tween 20, a water-soluble nonionic surfactant. Woodward et al. (2010b) prepared oil-in-water emulsions stabilized with sodium caseinate and treated the emulsion with transglutaminase to catalyze the formation of cross-links between protein molecules. They found that the rate of lipolysis in simulated duodenal conditions decreased significantly as a result of the transglutaminase-induced cross-linking of protein molecules. Their results suggested that a cross-linked interfacial film of casein proteins would be more resistant to displacement by bile salts. Li et al. (2011) also succeeded in reducing the rate of lipolysis by adopting a layer-by-layer electrostatic deposition approach: the formation of a protein layer on the oil droplet surface, followed by the deposition of a secondary layer of an anionic polysaccharide, alginate, on top of the protein layer.

An alternative approach to enhancing the resistance of interfacial protein films to surfactant-induced competitive displacement is to conjugate proteins with polysaccharides that can self-assemble to form networks. Polysaccharides can be conjugated with whey proteins using the initial stage of Maillard reactions, forming covalent linkages between nucleophilic amine groups of proteins and reducing ends of polysaccharides (Nagasawa et al., 1996; Akhtar and Dickinson, 2003; Zhu et al., 2008). The protein moiety of the conjugate is then expected to enable the conjugate to adsorb to the interface because of its surface activity, and the polysaccharide moiety forms self-assembled networks at the interface. The additional polysaccharide network is seen to increase the mechanical strength of the interfacial film as a whole, and therefore its resistance against the expansion of interfacial surfactant domains. In contrast, conjugation with polysaccharides that do not form self-assembled networks would have little effect on surfactant-induced displacement behavior, because such polysaccharides are unlikely to strengthen interfacial films. The objective of this study was to investigate the effect of conjugation between whey proteins and network-forming polysaccharides on the surfactant-induced competitive displacement of whey proteins from the air-water interface. Gellan, a food-grade gelling polysaccharide, was conjugated with whey protein because its molecular structure and properties have been well characterized and reported in the literature (Chandrasekaran et al., 1992; Ikeda et al., 2004a, 2013).

\section{MATERIALS AND METHODS}

\section{Materials}

Whey protein isolate (BiPRO, lot JE 226-2-420) was supplied by Davisco Foods International Inc. (Le Sueur, $\mathrm{MN})$. The protein content of the dry powder was $>90 \%$ (wt/wt) according to the manufacturer's specifications. Polyoxyethylene (20) sorbitan monolaurate (Tween 20) was obtained from Sigma-Aldrich (St. Louis, MO). Deacylated gellan (Kelcogel, lot 1J1780A) was provided by CP Kelco (Atlanta, GA). Other chemicals used in this study were of analytical grade.

\section{Preparation of Whey Protein-Gellan Conjugates by Heat Treatment}

The protein and polysaccharide were conjugated via the Maillard reaction, induced by heating of the mixtures under controlled humidity (Akhtar and Dickinson, 2003, 2007). Whey protein isolate and gellan were dissolved separately in deionized water, and then mixed at a weight ratio of 1 part whey protein isolate to 2 parts gellan. The mixed solution was adjusted to $\mathrm{pH} 7.0$ and then lyophilized. The lyophilized sample was placed in a preheated desiccator containing a saturated solution of $\mathrm{KCl}$ to achieve a relative humidity of $79 \%$, and then it was incubated at $80^{\circ} \mathrm{C}$ for $2 \mathrm{~h}$. The heat-treated product was adjusted to $\mathrm{pH} 5.0$ and then centrifuged at $7,000 \times g$ for $50 \mathrm{~min}$ to precipitate heat-denatured whey proteins (Bund et al., 2012). The supernatant was adjusted to $\mathrm{pH}$ 7.0. Protein components in the supernatant (including protein-polysaccharide conjugates) were precipitated by adding ammonium sulfate to a final concentration of $5 \mathrm{M}$. The precipitate was dissolved in distilled water and then dialyzed for over $24 \mathrm{~h}$ using a Spectra/Pro 6 dialysis membrane with a molecular-weight cutoff of $50 \mathrm{kDa}$ (Spectrum, Rancho Dominguez, CA) and an approximately 40-fold volume 
of distilled water to remove remaining unreacted whey proteins. The dialyzed solution was lyophilized and stored dry at $4^{\circ} \mathrm{C}$ before use.

\section{Deposition of Whey Protein-Gellan Conjugate on Mica}

The whey protein-gellan conjugate was dissolved in distilled water to a gellan concentration of $1 \mu \mathrm{g} / \mathrm{mL}$ and was adjusted to $\mathrm{pH} 10$ using $0.1 \mathrm{M} \mathrm{NaOH}$. A $2-\mu \mathrm{L}$ drop was deposited onto a freshly cleaved mica surface and allowed to stand in a covered Petri dish at room temperature for at least $15 \mathrm{~min}$ until no liquid was visible on the mica. The air-dried sample was imaged in air using AFM.

\section{Surfactant-Induced Competitive Displacement}

The whey protein isolate or the whey protein-gellan conjugate was dissolved in distilled water to a concentration of $1 \mathrm{mg} / \mathrm{mL}$. A $100-\mu \mathrm{L}$ sample was then carefully spread onto the air-water interface formed on a polytetrafluoroethylene trough (KSV Instruments, Helsinki, Finland). Surface pressure was monitored using a balance (KSV Instruments) equipped with a Wilhelmy plate. The interfacial film was equilibrated for 3 to $4 \mathrm{~h}$ at room temperature until a constant surface pressure was observed. In some selected experiments, gellan was dissolved in distilled water to a concentration of $0.3 \%$ (wt/wt) and a 1-mL sample was injected into the subphase and incubated overnight (approximately $18 \mathrm{~h}$ ) at room temperature.

To induce competitive displacement, a 1-mL sample of an aqueous solution containing $1 \mathrm{~m} M$ Tween 20 was injected into the sub-phase. The injection caused an increase in surface pressure as the surfactant adsorbed to the interface. More Tween 20 was added as needed to reach the desired surface pressure. Interfacial films were sampled at various surface pressures using the Langmuir-Blodgett method (Mackie et al., 1999) by dipping a freshly cleaved mica sheet mounted perpendicularly to the interface in and out of the sub-phase through the interface at a speed of $8.4 \mathrm{~mm} / \mathrm{min}$. Due to the hydrophilic nature of the mica surface, interfacial films were deposited onto the mica sheet on the upward stroke only. The transferred film was allowed to air-dry and imaged in $n$-butanol using AFM.

\section{Atomic Force Microscopy}

Samples deposited on or transferred to mica sheets were imaged using a BioScope Catalyst atomic force microscope (Bruker Corporation, Santa Barbara, CA) operated in peak force tapping mode. The sample surface was scanned at a rate of $1 \mathrm{~Hz}$ in air using a $\mathrm{V}$-shaped silicon nitride cantilever with a nominal spring constant of $0.4 \mathrm{~N} / \mathrm{m}$ and a resonant frequency of approximately $70 \mathrm{kHz}$ (Bruker Corporation, Camarillo, CA) or in $n$ butanol using a $\mathrm{V}$-shaped silicon nitride cantilever with a nominal spring constant of $0.7 \mathrm{~N} / \mathrm{m}$ and a resonant frequency of approximately $150 \mathrm{kHz}$ (Bruker Corporation, Camarillo, CA). Topographical data were then generated based on the trace of the z-position of the cantilever at its maximum deflection and processed using a basic image processing commands, such as "Plain Fit" and "Flatten," in NanoScope Analysis software version 1.40 (Bruker Corporation, Santa Barbara, CA). To evaluate the interfacial area coverage by whey proteins, images were converted to binary using ImageJ software version 1.49 (National Institutes of Health, Bethesda, MD). The threshold was set at approximately $15 \%$ of the full scale. At least 3 images were analyzed at a given surface pressure to evaluate the interfacial area coverage by whey proteins. The thickness of the interfacial whey protein films was evaluated using a nonbinarized image. At least 3 cross-sections of each image were arbitrarily selected to determine height profiles. The film thickness was defined as the height difference between the top surface of an interfacial film and that of the nearest mica substrate.

\section{Statistical Analysis}

Experiments were performed in triplicate to report means and standard deviations. The LSD between values for coverages and thicknesses of interfacial whey protein films were evaluated using independent $t$-tests and Fisher's LSD tests in R software version 2.15.1 (R Foundation for Statistical Computing, Vienna, Austria). $P$-values $<0.05$ were considered statistically significant.

\section{RESULTS AND DISCUSSION}

Figure 1 shows the progressive displacement of whey proteins from the air-water interface induced by the addition of Tween 20 and in the presence of gellan in the sub-phase. These interfacial whey protein films were prepared by adding an aqueous solution of whey proteins to the interface. Surface pressure continued to increase gradually for 3 to $4 \mathrm{~h}$ after the addition of whey proteins until it reached a constant value of $12 \mathrm{mN} / \mathrm{m}$. An aqueous solution of gellan was then injected into the subphase and incubated overnight. The addition of gellan to the sub-phase did not cause any significant changes to the surface pressure. A topographical AFM image of the interfacial whey protein film transferred onto mica at this stage, before the addition of surfactants (Figure 


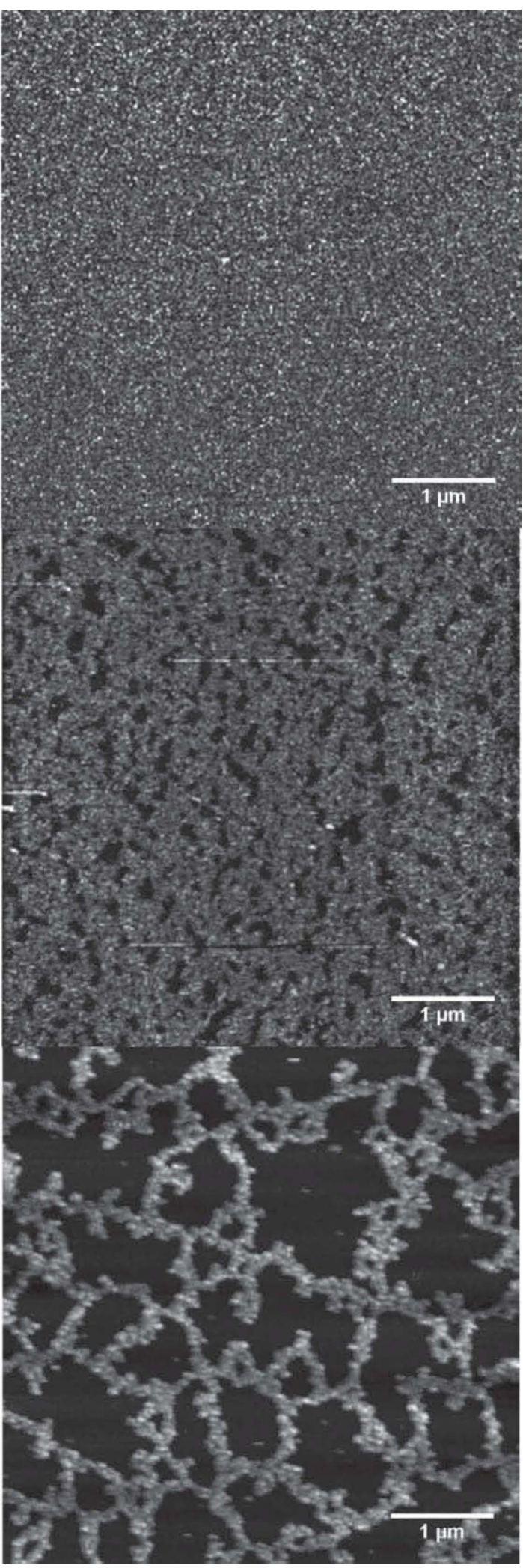

Figure 1. Topographical images of interfacial whey protein isolate films being displaced by Tween 20 in the presence of gellan in the sub-phase. The films were transferred onto mica sheets at a surface pressure of 12 (top), 23 (middle), or $28 \mathrm{mN} / \mathrm{m}$ (bottom). 1a), shows no network structures characteristic of the network-forming polysaccharide (Ikeda et al., 2004a, 2013). Because gellan has a glucuronic acid residue in its repeating unit (Chandrasekaran et al., 1992), it may bind electrostatically to positively charged groups of protein molecules. However, the present results suggest that gellan does not adsorb to the interfacial whey protein film in the examined conditions. Figures $1 \mathrm{~b}$ and $1 \mathrm{c}$ show the effect of the competitive adsorption of Tween 20 to the interfacial whey protein film. The adsorption of surfactants to the interface reduced the local surface tension and increased the difference in surface pressure between surfactant and protein domains, leading to the compression of the protein domain. Figure $1 \mathrm{~b}$ shows numerous irregularly shaped small holes where proteins were displaced by surfactants at a surface pressure of 23 $\mathrm{mN} / \mathrm{m}$, indicating that the strength of protein-protein interactions in the protein film was not uniform and that mechanically weaker points failed earlier (Mackie et al., 1999). Figure 1c shows that at a surface pressure of $28 \mathrm{mN} / \mathrm{m}$, protein domains were compressed to string-like structures.

Figure 2 shows changes in the interfacial area coverage by whey proteins during competitive displacement by Tween 20 in the presence and absence of gellan in the sub-phase. A slight reduction in the interfacial area coverage is noticeable at a surface pressure of $20 \mathrm{mN} / \mathrm{m}$, regardless of the presence of gellan in the sub-phase. Coverage decreases further with increasing surface pressure. We did not observe any significant effects of the presence of gellan in the sub-phase on the interfacial area coverage of whey proteins.

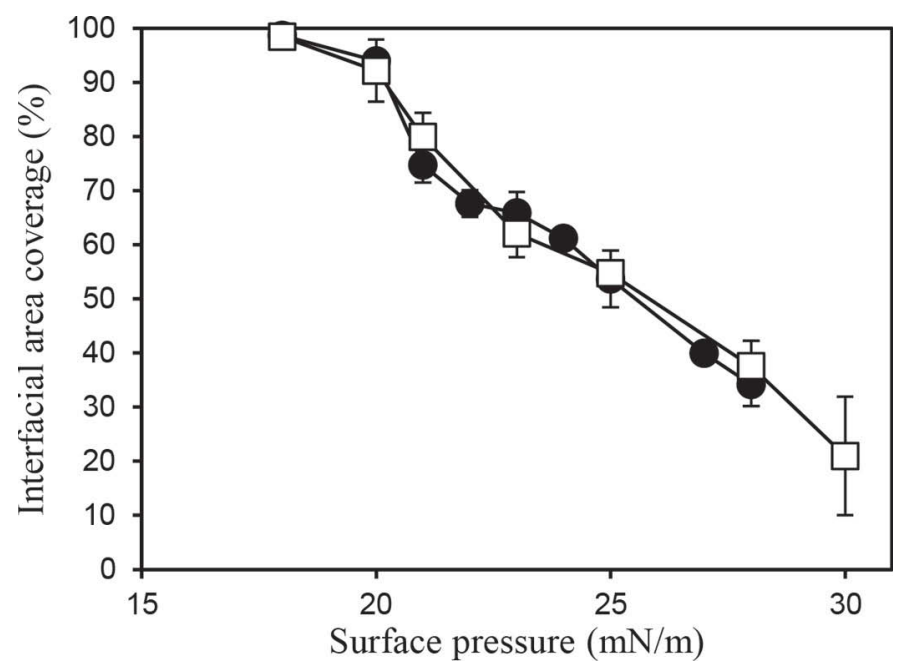

Figure 2. Changes in the interfacial area coverage by whey proteins during competitive displacement by Tween 20 in the presence (squares) and absence (circles) of gellan in the sub-phase. Error bars represent SD. 
Figure 3 shows increases in the thickness of interfacial whey protein films with increasing surface pressure. In the absence of gellan in the sub-phase, the film thickness increased gradually at first from approximately 1.3 $\mathrm{nm}$ at a surface pressure of $21 \mathrm{mN} / \mathrm{m}$ to $1.8 \mathrm{~nm}$ at $24 \mathrm{mN} / \mathrm{m}$, then more steeply to $5.2 \mathrm{~nm}$ at $28 \mathrm{mN} / \mathrm{m}$, and finally to $6.9 \mathrm{~nm}$ at $29 \mathrm{mN} / \mathrm{m}$. The presence of gellan in the sub-phase had no significant effects on film thickness. The initial thickness, corresponding to the thickness of a whey protein monolayer, was significantly smaller than the size of whey protein monomers in the native state (e.g., approximately $4 \mathrm{~nm}$ for $\beta$-LG; Aymard et al., 1999). However, $\beta$-LG has been reported to occupy an interfacial area of approximately $20 \mathrm{~nm}^{2}$ per molecule as a result of partial unfolding (Gochev et al., 2013). Given that the volume of the molecule does not change, the thickness of a $\beta-\mathrm{LG}$ monomer at the interface is estimated to be approximately $1.7 \mathrm{~nm}$, agreeing well with the initial film thickness observed in this study. At relatively low surface pressures up to approximately $24 \mathrm{mN} / \mathrm{m}$, the film thickness changed little with increasing surface pressure, but the film area coverage decreased significantly (Figure 2). Further increases in surface pressure resulted in simultaneous decreases in area coverage and increases in film thickness. Mackie et al. (1999) have reported similar trends and suggested that the initial decrease in film area without significant increases in film thickness represented a reordering of protein molecules at the interface to achieve a higher packing density, and that in the latter process, some protein molecules desorbed from the interface but did not fully dissociate from the film itself.

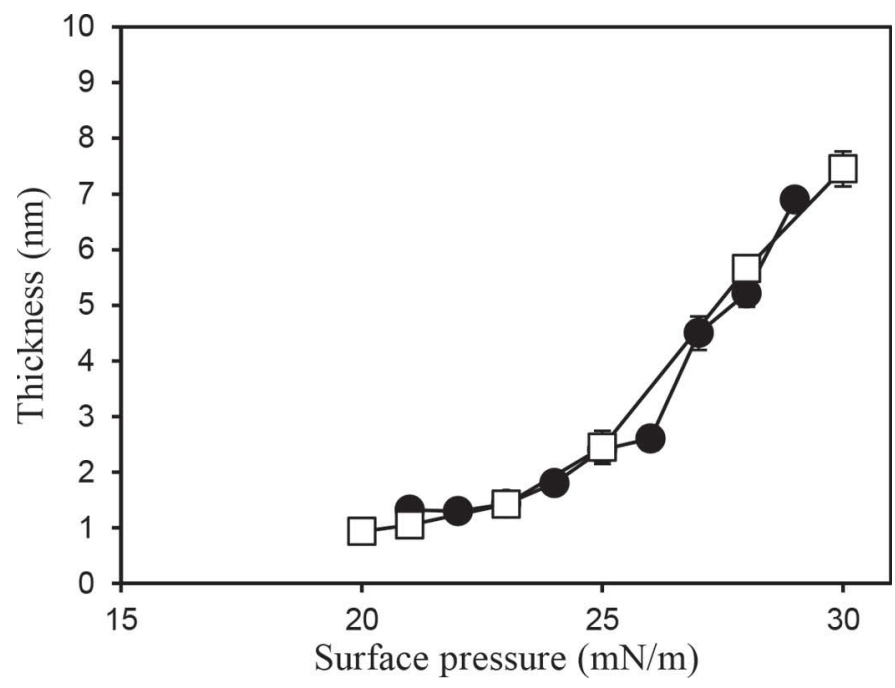

Figure 3. Changes in the thickness of interfacial whey protein films during competitive displacement by Tween 20 in the presence (squares) and absence (circles) of gellan in the sub-phase. Error bars represent SD.
We imaged interfacial films transferred onto the mica surface in butanol using AFM in accordance with the procedure reported in the literature (Mackie et al., 1999, 2001). Butanol dissolves surfactants and provides improved image contrasts, but it may induce some structural changes in interfacial protein films. Therefore, we also attempted imaging the interfacial films in air without the application of butanol. The film thickness obtained at a surface pressure of $28 \mathrm{mN} / \mathrm{m}$ was $6 \mathrm{~nm}$, slightly higher than the value determined in butanol $(5 \mathrm{~nm})$. These results indicate that interfacial whey protein film submerged in butanol may be compressed vertically, so that the film thickness is slightly underevaluated. Increases in film thickness are unlikely to be due to structural artifacts resulting from the application of butanol during AFM imaging.

The preceding sections have demonstrated that gellan molecules added to the sub-phase after the formation of interfacial whey protein films did not adsorb to the interfacial film, nor did they affect the surfactant-induced competitive displacement of whey proteins from the interface. Thus, we covalently linked whey proteins and gellan polysaccharides via the Maillard reaction to study the effect of this conjugation on surfactant-induced displacement of the whey proteingellan conjugate from the air-water interface. Figure 4a shows a topographical image of a whey protein-gellan conjugate dissolved in distilled water, adjusted to $\mathrm{pH}$ 10, deposited on mica, air-dried, and imaged in air using AFM. At pH 10, both whey protein and gellan are highly negatively charged. Any electrostatically bound complexes of whey protein and gellan would dissociate into individual components, should they exist. In the vicinity of the center of the image, a globular structure, presumably representing a single molecule of whey protein, can be seen. The height of the tallest point of the globular structure was determined to be approximately $6 \mathrm{~nm}$, slightly greater than the size of a monomer of native $\beta$-LG. It is therefore likely that the protein monomer swelled due to intramolecular electrostatic repulsions. It is also possible that the whey protein molecule fell on top of folded molecular chains of gellan upon its deposition on mica. The height of filamentous structures, presumably representing molecular chains of gellan, varied from approximately $0.8 \mathrm{~nm}$ to $1.5 \mathrm{~nm}$ as shown in the height profile beneath the image (Figure $4 \mathrm{~b})$. Such variability in height likely resulted from overlaps of gellan molecules. The contour length of gellan in the image was approximately $1,400 \mathrm{~nm}$ in total. This value corresponds to a molecular weight of approximately $500 \mathrm{kDa}$, assuming a molecular weight per unit contour length of $355 \mathrm{Da} / \mathrm{nm}$ (Ogawa et al., 2005). Using this value, the molar ratio of protein to gellan at the time of the conjugation reaction was estimated to 


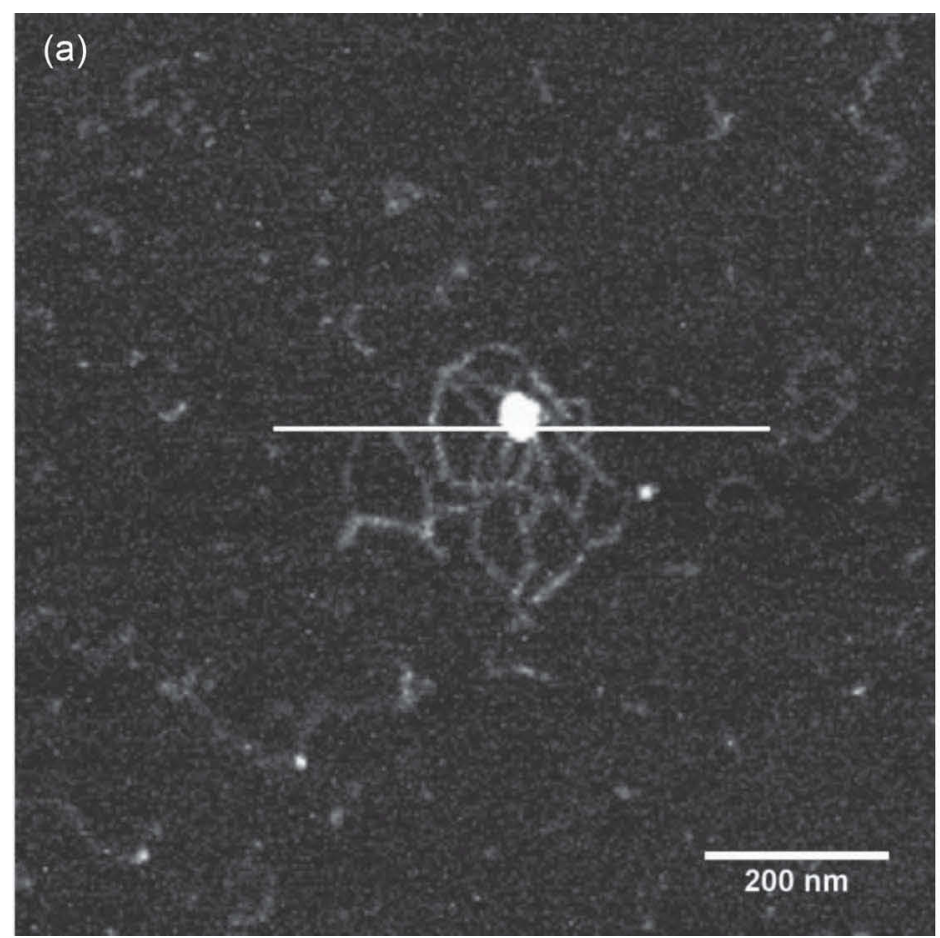

(b)

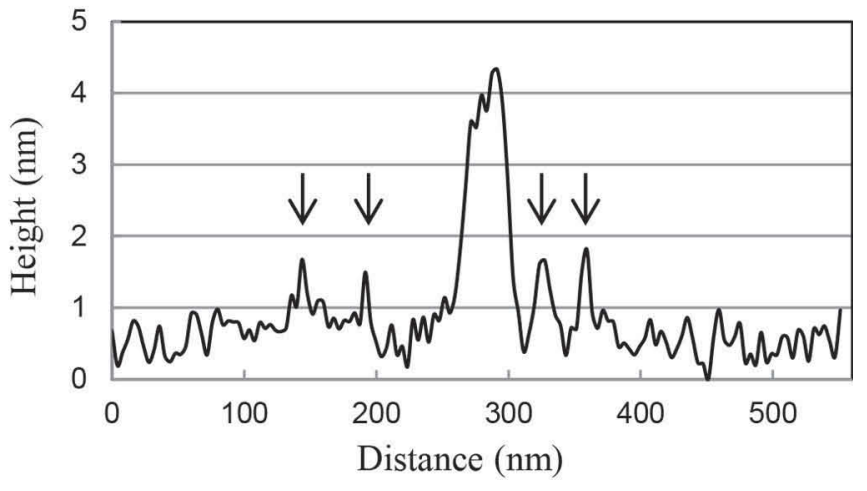

Figure 4. (a) Topographical image of whey protein-gellan conjugates deposited from an aqueous solution adjusted to $\mathrm{pH} 10$. (b) Height profile of the cross-section highlighted in (a). The arrows indicate molecular chains of gellan.

be approximately 14 to 1 . It is therefore likely that the majority of whey proteins remained unconjugated with the polysaccharides.

Whey protein-gellan conjugates were spread onto the air-water interface and then displaced by the addition of Tween 20. The addition of surfactants led to an immediate increase in surface pressure. Figure 5 shows a topographical image of an interfacial film of whey protein-gellan conjugates transferred onto mica at a surface pressure of $18 \mathrm{mN} / \mathrm{m}$. It appears that whey proteins occupied the entire interfacial area, but surfactant domains were too small to be noticeable. Filamentous structures were most likely to represent gellan polysaccharides. The contour length of the filamentous structure varied from several hundred $\mathrm{nm}$ to over $1 \mu \mathrm{m}$. The measured cross-sectional height of the gellan filaments was approximately $1.5 \mathrm{~nm}$ (Figure 5b), suggesting that these filamentous structures formed localized networks at the interface through inter-helical association between gellan molecules in the double-stranded helical conformation (Yuguchi et al., 1999; Ikeda et al., 2004b).

Dark domains of irregular shapes and various sizes, representing interfacial regions occupied by surfactants, emerged when the surface pressure increased to 23 $\mathrm{mN} / \mathrm{m}$ (Figure 6a). The observed variability in shape and size of surfactant domains was qualitatively consis- tent with the results observed for unconjugated whey proteins (Figure 1), indicating that protein-protein interactions at the interface were essentially unchanged by conjugation with gellan. The interfacial area coverage by whey proteins decreased to $70 \%$ at this surface pressure, and the film thickness increased to $1.8 \mathrm{~nm}$. The cross-sectional height of gellan filaments was approximately $1.8 \mathrm{~nm}$. This value did not change significantly with increasing surface pressure. The magnifications of the boxed areas highlighted in Figure 6a (inset: Figures $6 \mathrm{~b}$ and $6 \mathrm{c}$ ) show certain heterogeneity in the structure of the interfacial film: the coverage of whey proteins in the interfacial regions where gellan networks are present (70\% in Figure 6c) was greater than that in the interfacial regions where no gellan networks can be seen $(66 \%$ in Figure $6 \mathrm{~b})$. These results indicate that whey protein-gellan conjugates were more resistant to surfactant-induced competitive displacement from the interface than whey protein alone. They also indicate that gellan networks formed at the interface increased the mechanical strength of interfacial films to provide resistance against lateral compressional forces exerted by surface pressure differences between surfactant and protein domains.

Interfacial regions where gellan networks are present should contain whey protein-gellan conjugates, 
but those where gellan networks are absent should not. Figure 7 shows comparisons of the interfacial area coverage by whey proteins between conjugate-containing and conjugate-lacking interfacial regions. The area coverage started to decrease at a surface pressure of 18 $\mathrm{mN} / \mathrm{m}$ in conjugate-lacking regions, and at $20 \mathrm{mN} / \mathrm{m}$ in conjugate-containing regions. At a given surface pressure, conjugate-containing regions showed a greater area coverage than conjugate-lacking regions. As surface pressure increased to $25 \mathrm{mN} / \mathrm{m}$, the difference in the area coverage became statistically significant $(P<$ $0.05)$. These results confirmed that unconjugated whey proteins are more susceptible to surfactant-induced displacement. At a relatively high surface pressure (e.g., $28 \mathrm{mN} / \mathrm{m}$ ), interfacial regions occupied by unconjugated whey proteins appeared to diminish (Figure 8), leaving domains occupied by surfactants and domains occupied by conjugates only. We found qualitative differences in the network structure of the film containing whey protein only (Figure 1c) and the conjugate film (Figure 8) at an identical surface pressure of $28 \mathrm{mN} / \mathrm{m}$. The width of the strands in the former appeared to correspond to several molecular diameters of whey proteins, but the strand width in the latter appeared to be approximately equal to the molecular diameter of whey proteins. Although the exact reasons for this were not readily discernable, the gellan moiety of the conjugate seemed to play a significant role in the protein network structure.

Figure 9 shows the thickness of interfacial films in conjugate-containing regions compared with that in conjugate-lacking regions. The thickness in both regions increased gradually at first, and then in a more pronounced way as surface pressure increased beyond approximately $23 \mathrm{mN} / \mathrm{m}$. Interfacial films in conjugatecontaining regions seemingly had greater area coverages than those in conjugate-lacking regions. However, the mean values of film thicknesses did not show significant differences at surface pressures of 20 to $25 \mathrm{mN} / \mathrm{m}$. We were unable to collect thickness data for conjugatelacking interfacial regions at surface pressures $>25$ $\mathrm{mN} / \mathrm{m}$ because of the disappearance of such regions. The thickness of interfacial films of whey protein-gellan conjugates increased to approximately $4.5 \mathrm{~nm}$ as surface pressure increased to $28 \mathrm{mN} / \mathrm{m}$. The increase in the thickness of interfacial films of conjugates and the concurrent reduction in film area were qualitatively consistent with the orogenic displacement model (Mackie et al., 1999). Interfacial films of whey protein alone and those of whey protein-gellan conjugates exhibited similar film thicknesses at a given surface pressure, but the latter had a slightly greater film area, suggesting

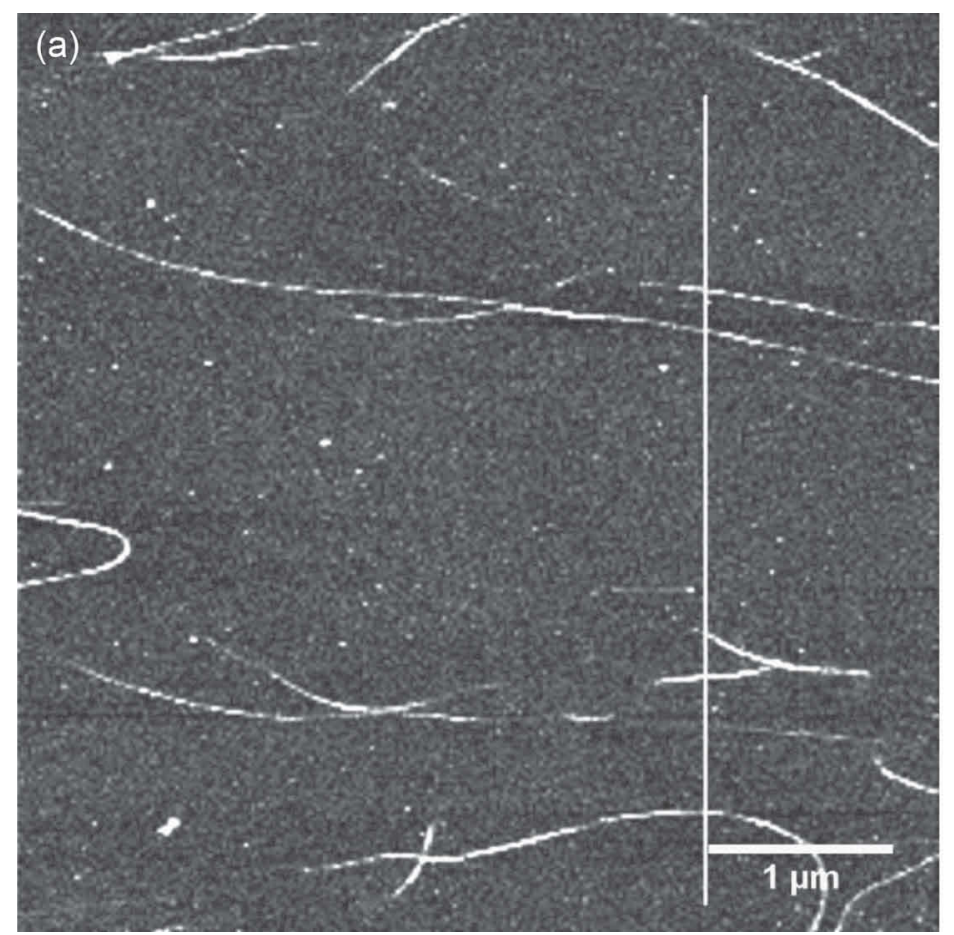

(b)

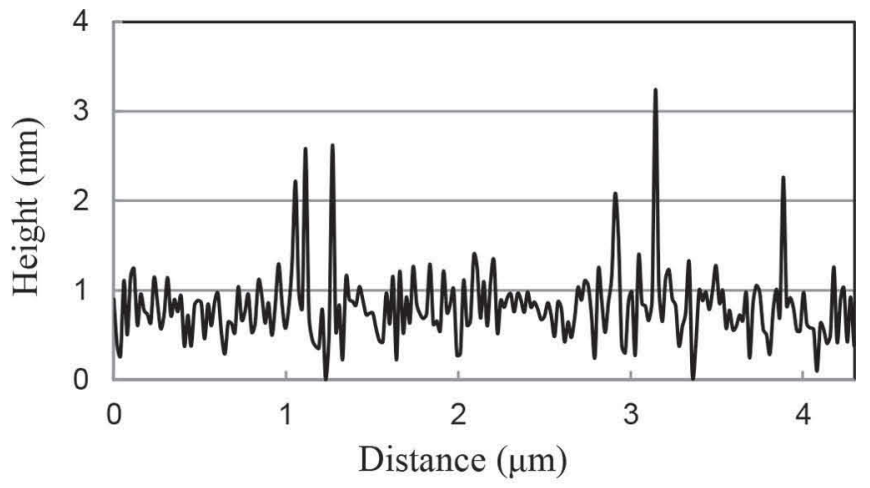

Figure 5. (a) Topographical image of whey protein-gellan conjugates being displaced by Tween 20. The film was transferred onto a mica sheet at a surface pressure of $18 \mathrm{mN} / \mathrm{m}$. (b) Height profile of the cross-section highlighted in (a). 


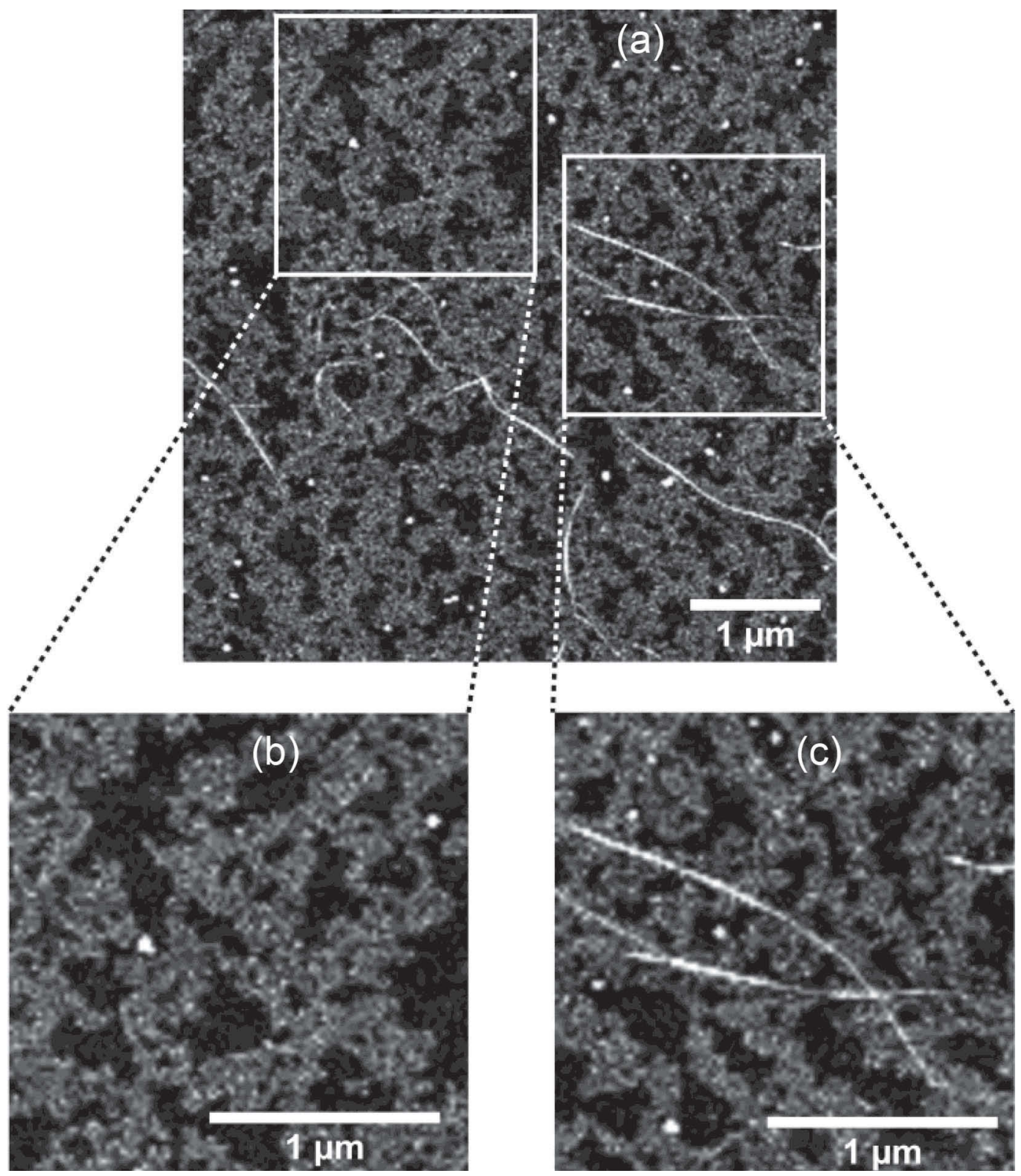

Figure 6. (a) Topographical image of whey protein-gellan conjugates being displaced by Tween 20 . The film was transferred onto a mica sheet at a surface pressure of $23 \mathrm{mN} / \mathrm{m}$. (b) and (c) Magnified images of the boxed areas in (a).

that gellan networks present at the interface enhanced the resistance of interfacial protein films to the expansion of surfactant domains.

\section{CONCLUSIONS}

Whey protein-gellan conjugates demonstrated increased resistance against surfactant-induced displacement from the air-water interface. Gellan added to the sub-phase after the formation of a monolayer of whey proteins at the air-water interface did not adsorb to the monolayer, but whey protein-gellan conjugates did adsorb to the interface, enabling the formation of selfassembled networks of gellan. The majority of whey protein molecules adsorbed to the interface were not conjugated with gellan under the reaction conditions used in this study. Therefore, gellan networks formed at the interface were localized. These results suggest that the efficiency of the conjugation reaction was relatively low, presumably because of a low molar ratio of reducing ends of polysaccharides available to reactive amine groups of proteins during the reaction. The next step of 


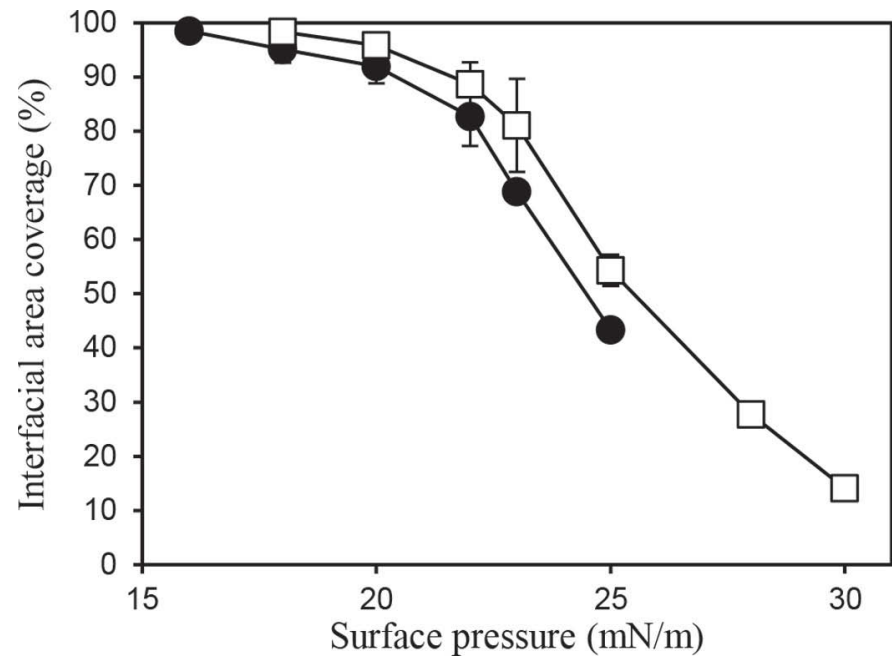

Figure 7. Changes in the interfacial area coverage by unconjugated whey proteins (squares) and whey protein-gellan conjugates (circles) during competitive displacement by Tween 20. Error bars represent SD.

investigation should involve efforts to further improve resistance against surfactant-induced displacement by increasing the interfacial concentration of conjugates. Nevertheless, the interfacial area coverage by whey protein-gellan conjugates was greater than that of whey

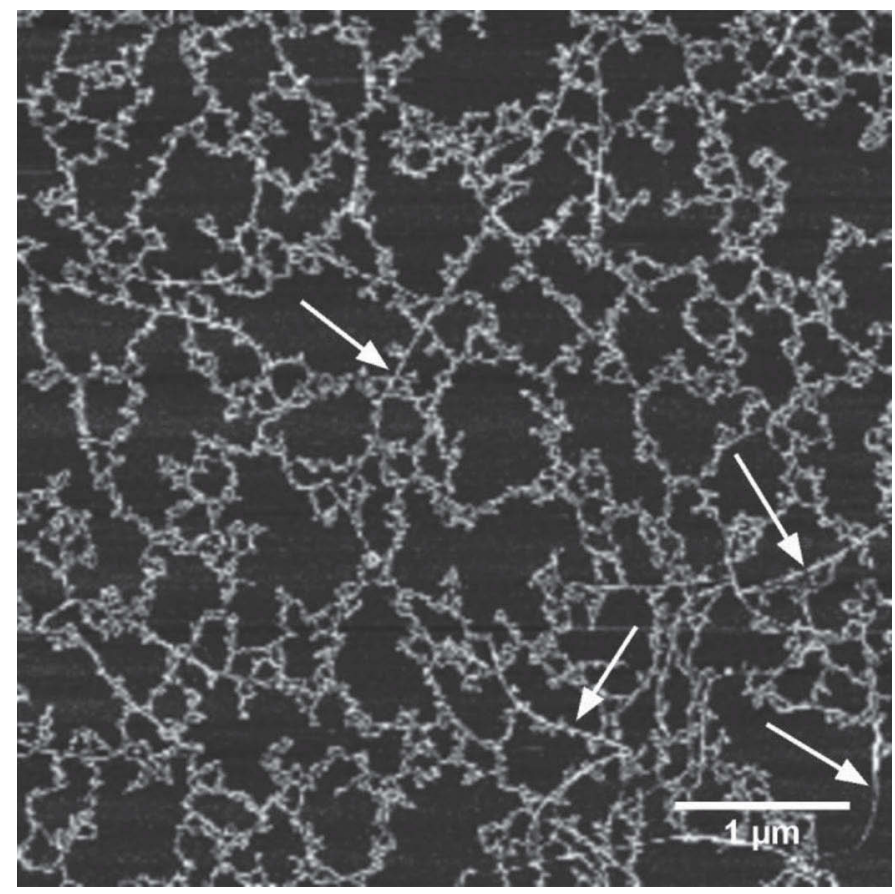

Figure 8. Topographical image of whey protein-gellan conjugates being displaced by Tween 20 . The film was transferred onto a mica sheet at a surface pressure of $28 \mathrm{mN} / \mathrm{m}$. The arrows indicate gellan molecules.

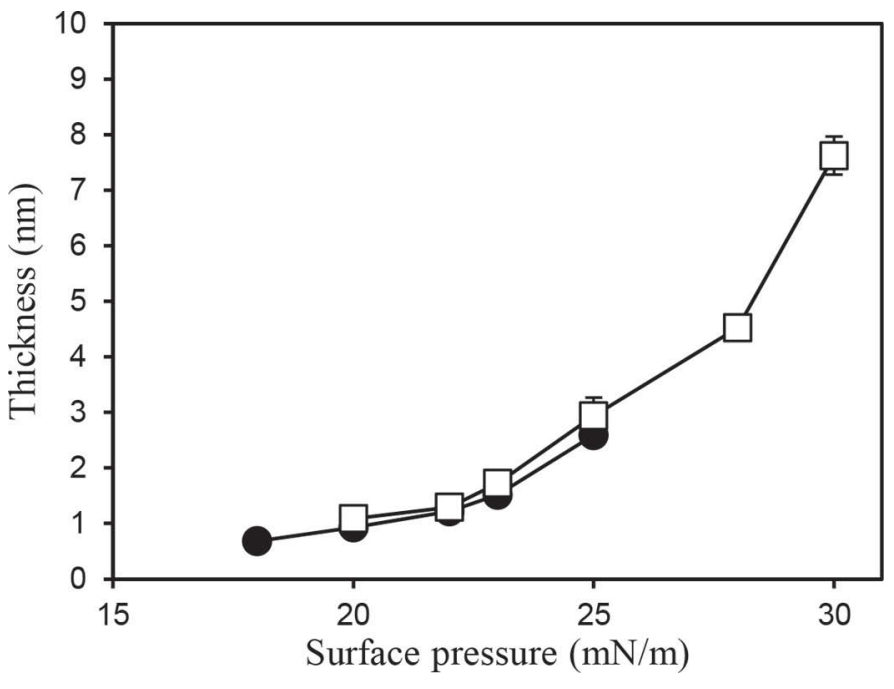

Figure 9. Changes in the thickness of interfacial films of unconjugated whey proteins (squares) and whey protein-gellan conjugates (circles) during competitive displacement by Tween 20. Error bars represent SD.

protein alone at high surface pressures induced by surfactant, confirming that the conjugate was more resistant to desorption and displacement. This resistance was most likely because of the formation of additional networks of gellan in the whey protein-gellan film adsorbed to the air-water interface.

\section{ACKNOWLEDGMENTS}

We thank Davisco Foods International, Inc. (Le Sueur, MN) and CP Kelco (Atlanta, GA) for kindly providing whey protein isolate and gellan samples, respectively. We also thank Srinivasan Damodaran for valuable discussions and letting us use his laboratory equipment. This work was partially supported by the USDA National Institute of Food and Agriculture (Washington, DC; Hatch project 1003055).

\section{REFERENCES}

Akhtar, M., and E. Dickinson. 2003. Emulsifying properties of whey protein-dextran conjugates at low $\mathrm{pH}$ and different salt concentrations. Colloids Surf. B Biointerfaces 31:125-132.

Akhtar, M., and E. Dickinson. 2007. Whey protein-maltodextrin conjugates as emulsifying agents: An alternative to gum arabic. Food Hydrocoll. 21:607-616.

Aymard, P., T. Nicolai, and D. Durand. 1999. Static and dynamic scattering of $\beta$-lactoglobulin aggregates formed after heat-induced denaturation at $\mathrm{pH}$ 2. Macromolecules 32:2542-2552.

Bund, T., S. Allelein, A. Arunkumar, J. A. Lucey, and M. R. Etzel. 2012. Chromatographic purification and characterization of whey protein-dextran glycation products. J. Chromatogr. A 1244:98 105.

Chandrasekaran, R., A. Radha, and V. G. Thailambal. 1992. Roles of potassium ions, acetyl and L-glyceryl groups in native gellan double helix: An X-ray study. Carbohydr. Res. 224:1-17. 
Damodaran, S. 2005. Protein stabilization of emulsions and foams. J. Food Sci. 70:R54-R66.

De Feijter, J. A., J. Benjamins, and M. Tamboer. 1987. Adsorption displacement of proteins by surfactants in oil-in-water emulsions. Colloids Surf. 27:243-266.

Dickinson, E. 2001. Milk protein interfacial layers and the relationship to emulsion stability and rheology. Colloids Surf. B Biointerfaces 20:197-210.

Dickinson, E., and S. T. Hong. 1994. Surface coverage of $\beta$-lactoglobulin at the oil-water interface: Influence of protein heat treatment and various emulsifiers. J. Agric. Food Chem. 42:1602-1606.

Gochev, G., I. Retzlaff, E. V. Aksenenko, V. B. Fainerman, and R. Miller. 2013. Adsorption isotherm and equation of state for $\beta$-lactoglobulin layers at the air/water interface. Colloids Surf. A Physicochem. Eng. Asp. 422:33-38.

Ikeda, S., S. Gohtani, K. Nishinari, and Q. Zhong. 2013. High acyl gellan networks probed by rheology and atomic force microscopy. Food Sci. Technol. Res. 19:201-210.

Ikeda, S., Y. Nitta, B. S. Kim, T. Temsiripong, R. Pongsawatmanit, and K. Nishinari. 2004b. Single-phase mixed gels of xyloglucan and gellan. Food Hydrocoll. 18:669-675.

Ikeda, S., Y. Nitta, T. Temsiripong, R. Pongsawatmanit, and K. Nishinari. 2004a. Atomic force microscopy studies on cation-induced network formation of gellan. Food Hydrocoll. 18:727-735.

Li, Y., M. Hu, Y. M. Du, and D. J. McClements. 2011. Controlling lipid nanoemulsion digestion using nanolaminated biopolymer coatings. J. Microencapsul. 28:166-175.

Mackie, A. R., A. P. Gunning, M. J. Ridout, P. J. Wilde, and V. J. Morris. 2001. Orogenic displacement in mixed $\beta$-lactoglobulin/ $\beta$ casein films at the air/water interface. Langmuir 17:6593-6598.

Mackie, A. R., A. P. Gunning, P. J. Wilde, and V. J. Morris. 1999. Orogenic displacement of protein from the air/water interface by competitive adsorption. J. Colloid Interface Sci. 210:157-166.
Morris, V. J., N. C. Woodward, and A. P. Gunning. 2011. Atomic force microscopy as a nanoscience tool in rational food design. J. Sci. Food Agric. 91:2117-2125.

Nagasawa, K., K. Takahashi, and M. Hattori. 1996. Improved emulsifying properties of $\beta$-lactoglobulin by conjugating with carboxymethyl dextran. Food Hydrocoll. 10:63-67.

Ogawa, E., R. Takahashi, H. Yajima, and K. Nishinari. 2005. Thermally induced coil-to-helix transition of sodium gellan gum with different molar masses in aqueous salt solutions. Biopolymers 79:207-217.

Roth, S., B. S. Murray, and E. Dickinson. 2000. Interfacial shear rheology of aged and heat-treated $\beta$-lactoglobulin films: Displacement by nonionic surfactant. J. Agric. Food Chem. 48:1491-1497.

Woodward, N. C., A. P. Gunning, J. Maldonado-Valderrama, P. J. Wilde, and V. J. Morris. 2010a. Probing the in situ competitive displacement of protein by nonionic surfactant using atomic force microscopy. Langmuir 26:12560-12566.

Woodward, N. C., A. P. Gunning, P. J. Wilde, B.-S. Chu, and V. J. Morris. 2010b. Engineering interfacial structures to moderate satiety. Pages 367-376 in Gums and Stabilisers for the Food Industry. Vol. 15. P. A. Williams and G. O. Phillips, ed. The Royal Society of Chemistry, Cambridge, UK.

Woodward, N. C., P. J. Wilde, A. R. Mackie, A. P. Gunning, P. A Gunning, and V. J. Morris. 2004. Effect of processing on the displacement of whey proteins: Applying the orogenic model to a real system. J. Agric. Food Chem. 52:1287-1292.

Yuguchi, Y., H. Urakawa, S. Kitamura, I. Wataoka, and K. Kajiwara. 1999. The sol-gel transition of gellan gum aqueous solutions in the presence of various metal salts. Prog. Colloid Polym. Sci. 114:4147.

Zhu, D., S. Damodaran, and J. A. Lucey. 2008. Formation of whey protein isolate (WPI)-dextran conjugates in aqueous solutions. J. Agric. Food Chem. 56:7113-7118. 